\title{
A NOTE ON COMPLEMENTARY SUBSPACES IN A RIEMANNIAN SPACE
}

\author{
YUNG-CHOW WONG ${ }^{1}$
}

\section{Introduction. ${ }^{2}$ Let}

$$
x^{\kappa}=x^{\kappa}\left(u^{a}\right), \quad \kappa, \lambda, \mu, \nu=1, \cdots, n ; a, b, \cdots, f=1, \cdots, m,
$$

be the equations of a $V_{m}$ in a $V_{n}$ with fundamental tensor $g_{\lambda \kappa}$ and let

$$
B_{a}^{\kappa}=\partial_{a} x^{\kappa} \equiv \frac{\partial x^{\kappa}}{\partial u^{a}} .
$$

Then the fundamental tensor and curvature tensor of $V_{m}$ in $V_{n}$ are, respectively,

$$
\begin{aligned}
& { }^{\prime} g_{c b}=g_{\lambda \kappa} B_{c}^{\lambda} B_{b}^{\kappa}, \\
& H_{c b}^{* \kappa}=D_{c} B_{b}^{\kappa} \equiv \partial_{c} B_{b}^{\kappa}+-\Gamma_{\mu \lambda}^{\kappa} B_{c}^{\mu} B_{b}^{\lambda}-{ }^{\prime} \Gamma_{c b}^{a} B_{a}^{\kappa},
\end{aligned}
$$

where $D$ denotes the generalized covariant differentiation with respect to $V_{m}$ in $V_{n}$; and $\Gamma_{\mu \lambda}^{\alpha}$ and ' $\Gamma_{c b}^{a}$ are, respectively, the Christoffel symbols of the second kind for $V_{n}$ and $V_{m}$.

By definition a $V_{m}$ in $V_{n}$ is said to be totally semi-umbilical in $V_{n}$ if a vector $v_{\kappa}$ exists such that

$$
v_{\kappa} \ddot{H_{c b} \kappa}={ }^{\prime} g_{c b}
$$

is satisfied at every point of $V_{m}$. In particular, this condition is evidently fulfilled when $H_{c b}^{* \kappa}$ has the form $H_{c b}^{\cdot * \kappa}={ }^{\prime} g_{c b} n^{\kappa}, n^{\kappa}$ being a certain vector; in this case we call $V_{m}$ totally umbilical in $V_{n}$.

In what follows we shall consider the subspaces $V_{m}: x^{p}=$ const. in a $V_{n}$ with fundamental tensor of the form

$$
g_{\lambda \kappa}=\left(\begin{array}{cc}
g_{c b} & 0 \\
0 & g_{q p}
\end{array}\right), \quad \begin{array}{r}
a, b, \cdots, f=1, \cdots, m, \\
p, q, \cdots, s=m+1, \cdots, n .
\end{array}
$$

Received by the editors, April 22, 1942.

1 The author is a Chinese Ying-Keng Funds student. He wishes to thank Professor D. J. Struik for the conversations they had from time to time during his stay at Cambridge, Massachusetts.

${ }^{2}$ For the theory of subspaces $V_{m}$ in a Riemannian $n$-space $V_{n}$, see SchoutenStruik, Einfiuhrung in der neuern Methoden der Differentialgeometrie II, Groningen, 1938 chap. 3.

${ }^{3} \mathrm{D}$. Perepelkine, Sur la courbure et les espaces normaux d'une $V_{m}$ dans $R_{n}$, Rec. Math. (Mat. Sbornik) N.S. vol. 42 (1935) pp. 81-100. 
The two families of subspaces $V_{m}: x^{p}=$ const. and $V_{n-m}: x^{a}=$ const. are called completementary families of subspaces in $V_{n}$. Recently, Yano ${ }^{4}$ proved that a condition for $V_{m}$ to be totally umbilical in $V_{n}$ is that $g_{c b}$ be of the form ${ }^{5} g_{c b}=\sigma\left(x^{\kappa}\right) \bar{g}_{c b}\left(x^{a}\right)$. We shall obtain, among other results, a similar condition for $V_{m}$ to be totally semi-umbilical in $V_{n}$.

2. First normal complex. Let $w^{a}$ and $y^{a}$ be two arbitrary vectors in $V_{m}$. Then the vector $y^{c} w^{b} H_{c b}^{* \kappa}$ spans the first normal complex of $V_{m}$ in $V_{n}$, whose dimensionality $m_{1}$ is therefore equal to the rank of the matrix $\left[H_{c b}^{\cdot * k}\right]$. In this matrix, as well as in every matrix appearing hereafter, $\kappa$ or $p$ indicates the column and the combination of $b, c, \cdots$ the row.

Now for the subspaces $V_{m}: x^{p}=$ const. in a $V_{n}$ with fundamental tensor (1.6), we have

$$
\begin{array}{rlrl}
B_{a}^{\kappa} & =\frac{\partial x^{\kappa}}{\partial x^{a}}=\delta_{a}^{\kappa}, & ' g_{c b}=g_{c b}, \\
H_{c b}^{* a}=\Gamma_{c b}^{a}-{ }^{\prime} \Gamma_{c b}^{a}, & H_{c b}^{* p p}=\Gamma_{c b}^{p} .
\end{array}
$$

But from (1.6) and the definition of the Christoffel symbols of the second kind

$$
\Gamma_{\mu \lambda}^{\kappa}=(1 / 2) g^{\kappa \nu}\left(\partial_{\mu} g_{\nu \lambda}+\partial_{\lambda} g_{\nu \mu}-\partial_{\nu} g_{\mu \lambda}\right)
$$

it follows at once that

$$
\begin{array}{ll}
\Gamma_{c b}^{a}={ }^{\prime} \Gamma_{c b}^{a}, & \Gamma_{c b}^{p}=-(1 / 2) g^{p q} \partial_{q} g_{c b}, \\
\Gamma_{c q}^{a}=(1 / 2) g^{a b} \partial_{q} g_{c b}, & \Gamma_{c r}^{p}=(1 / 2) g^{p q} \partial_{c} g_{r q} .
\end{array}
$$

Thus (2.2) become

$$
H_{c b}^{\cdot a}=0, \quad \because \cdot H_{c b}=-(1 / 2) g^{p q} \partial_{q} g_{c b} .
$$

And therefore the dimensionality $m_{1}$ of the first normal complex of $V_{m}$ in $V_{n}$ is equal to the rank of the matrix $\left[g^{p q} \partial_{q} g_{c b}\right]$. Since Det $\left(g^{p q}\right) \neq 0, m_{1}$ is also the rank of the matrix $\left[\partial_{p} g_{c b}\right]$. Hence ${ }^{6}$ there

${ }^{4} \mathrm{~K}$. Yano, Conformally separable quadratic differential forms, Proc. Imp. Acad. Tokyo vol. 16 (1940) pp. 83-86. For $n=m+1$ see L. P. Eisenhart, Riemannian geometry, Princeton, 1926 p. 182.

${ }^{5}$ Throughout this paper we denote by $\rho, \sigma, \theta, \phi$ scalar functions of $x^{k}$.

' See, for example, T. Levi-Civita, The absolute differential calculus, London, 1927 pp. 9-12. 
exist $m_{1}$ components of $g_{c b}$, which are functionally independent with regard to $x^{p}$, such that each component of $g_{c b}$ is expressible in terms of them and $x^{a}$. Conversely, it is evident from (2.4) that if $g_{c b}$ has this property, the first normal complex of $V_{m}$ in $V_{n}$ is of dimension $m_{1}$. Hence we have this theorem. ${ }^{7}$

THEOREM 2.1. The first normal complex of the subspaces $x^{p}=$ const. in a $V_{n}$ with fundamental tensor (1.6) is of dimension $m_{1}$, if and only if the matrix $\left[\partial_{p} g_{c b}\right]$ is of rank $m_{1}$, that is if $g_{c b}$ is of the form $g_{c b}=g_{c b}\left(x^{a}, \rho_{1}, \cdots, \rho_{m_{1}}\right)$, where the $\rho$ 's are $m_{1}$ functions of $x^{k}$ which are functionally independent with regard to $x^{p}$.

Now it follows from (2.4) and Theorem 2.1 that the components of the vector $y^{c} w^{b} H_{c b}^{-k}$, which spans the first normal complex, are

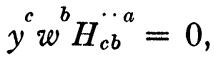

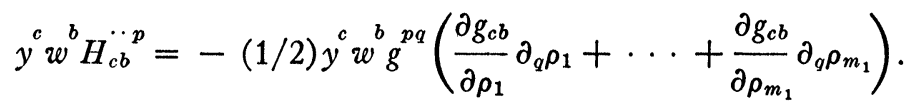

To see the implication of these equations let us consider a certain fixed $V_{n-m}: x^{a}=x_{0}^{a}$. Each $V_{m}$ of the family $x^{p}=$ const. has a point in common with $V_{n-m}$, at which the first normal complex of $V_{m}$ lies in the tangent space of $V_{n-m}$. Equations (2.5) then show that these first normal complexes are orthogonal to the subspaces

$$
\rho_{1}\left(\stackrel{a}{x_{0}}, x^{p}\right)=\text { const., } \cdots, \rho_{m_{1}}\left(\underset{x_{0}}{a}, x^{p}\right)=\text { const. }
$$

of $V_{n-m}$.

THEOREM 2.2. If a $V_{n}$ admits two complementary families of $V_{m}$ and $V_{n-m}$, then the first normal complexes, dimensionality $m_{1}$, of $V_{m}$ at points of any fixed $V_{n-m}$ are orthogonal to a family of subspaces $V_{n-m-m_{1}}$ in $V_{n-m}$.

The condition for $V_{m}$ to be minimal in $V_{n}$ is ${ }^{\prime} g^{c b} H_{c b}^{* k}=0$, which, by (2.1) and (2.4), can be written $g^{c b} \partial_{p} g_{c b}=0$, that is, $\partial_{p}$ Det $\left(g_{c b}\right)=0$. Hence this theorem follows. ${ }^{8}$

THEOREM 2.3. If a $V_{n}$ admits two complementary families of $V_{m}$ and $V_{n-m}$, a necessary and sufficient condition for $V_{m}$ to be minimal in $V_{n}$ is that $V_{n-m}$ determine a correspondence between them which preserves volume.

${ }^{7}$ For $m_{1}=0$, see Eisenhart, loc. cit. p. 186 Example 13.

${ }^{8}$ For $n=m+1$, see Eisenhart, loc. cit. p. 179. 
3. Totally semi-umbilical $V_{m}$. According to (1.5), (2.1) and (2.4), the condition for $x^{p}=$ const. to be totally semi-umbilical in $V_{n}$ is that a vector $v^{p}$ in $V_{n-m}$ exists such that $v^{p} \partial_{p} g_{c b}=g_{c b}$, that is,

$$
v^{p} \partial_{p} \log g_{c b}=1 \text {. }
$$

From this it follows that

$$
v^{p} \partial_{p} \log \left(g_{c b} / g_{e d}\right)=0 .
$$

Now if the first normal complex of $V_{m}$ in $V_{n}$ is of dimension $m_{1}$, then by Theorem $2.1 \mathrm{~g}_{c b}$ is of the form

$$
g_{c b}=g_{c b}\left(x^{a}, \rho_{1}, \cdots, \rho_{m_{1}}\right) .
$$

Consequently, (3.2) gives

$$
\theta_{1} \frac{\partial}{\partial \rho_{1}} \log \left(g_{c b} / g_{e d}\right)+\cdots+\theta_{m_{1}} \frac{\partial}{\partial \rho_{m_{1}}} \log \left(g_{c b} / g_{e d}\right)=0
$$

where

$$
\theta_{1}=\tau^{p} \partial_{p} \rho_{1}, \cdots, \theta_{m_{1}}=v^{p} \partial_{p} \rho_{m_{1}} .
$$

Conversely, let $m_{1}$ functions $\theta_{1}, \cdots, \theta_{m_{1}}$ exist satisfying (3.4). Then since $\rho_{1}, \cdots, \rho_{m_{1}}$ are independent with regard to $x^{p}$, the matrix $\left[\partial_{p} \rho_{1}, \cdots, \partial_{p} \rho_{m_{1}}\right]$ is of rank $m_{1}$. Therefore, the system of linear equations (3.5) has solutions for $v^{p}$; that is, $v^{p}$ exist satisfying (3.2) and also (3.1). Hence, when (3.3) is true, (3.4) is a necessary and sufficient condition for $V_{m}$ to be totally semi-umbilical in $V_{n}$.

On the other hand, by a well known theorem ${ }^{9}$ on the essential parameters of a set of functions, equation (3.4) is also the condition that there exist $m_{1}-1$ functions $\sigma_{1}, \cdots, \sigma_{m_{1}-1}$ of $x^{a}$ and the $\rho$ 's (and therefore of $x^{\kappa}$ ) such that $g_{c b} / g_{e d}$ is expressible in terms of them and $x^{a}$; that is, that $g_{c b}$ is of the form

$$
g_{c b}=\sigma_{m_{1}} \bar{g}_{c b}\left(x^{a}, \sigma_{1}, \cdots, \sigma_{m_{1}-1}\right) .
$$

It is seen that $\bar{g}_{c b}$ cannot be expressed in terms of $x^{a}$ and less than $m_{1}-1$ independent (with regard to $x^{p}$ ) functions $\sigma$ 's; otherwise, $g_{c b}$ would be expressible in terms of $x^{a}$ and less than $m_{1}$ functions, and consequently by Theorem 2.1 , the first normal complex of $V_{m}$ in $V_{n}$ would be of dimension less than $m_{1}$.

THEOREM 3.1. In a $V_{n}$ with fundamental tensor (1.6), each of the subspaces $x^{p}=$ const., whose first normal complexes are of dimension

${ }^{9}$ See, for example, L. P. Eisenhart, Continuous groups of transformation, Princeton, 1933, p. 9. 
$m_{1}$, is totally semi-umbilical in $V_{n}$, if and only if $g_{c b}$ is of the form

$$
g_{c b}=\sigma_{m_{1}} \bar{g}_{c b}\left(x^{a}, \sigma_{1}, \cdots, \sigma_{m_{1}-1}\right),
$$

where the $\sigma$ 's are $m_{1}$ functions of $x^{\kappa}$ which are independent with regard to $x^{p}$.

For $m_{1}=1$, we have Yano's result quoted in $\$ 1$.

4. Normal complexes of higher order. We now return to the end of $\S 2$ and consider the matrix

$$
\left(\begin{array}{c}
B_{a}^{\kappa} \\
H_{c b}^{* \kappa} \\
D_{f} H_{e d}^{* \kappa}
\end{array}\right) .
$$

Let $m_{1}$ and $m_{2}$ be, respectively, the dimensionalities of the first and second normal complexes of $V_{m}$ in $V_{n}$, then the rank of the above matrix is $m+m_{1}+m_{2}$. Taking account of (2.3), (2.4) and

$$
D_{f} H_{e d}^{\cdots \kappa}=\partial_{f} H_{e d}^{\cdots \kappa}+\Gamma_{\mu \lambda}^{\kappa} H_{e d}^{\cdot \lambda} B_{f}^{\mu}-{ }^{\prime} \Gamma_{f d}^{c} H_{e c}^{\cdots \kappa}-{ }^{\prime \kappa} \Gamma_{f e}^{c} H_{c d}^{\cdots \kappa}
$$

we can easily prove from (4.1) that the following matrices are all of rank $m_{1}+m_{2}$ :

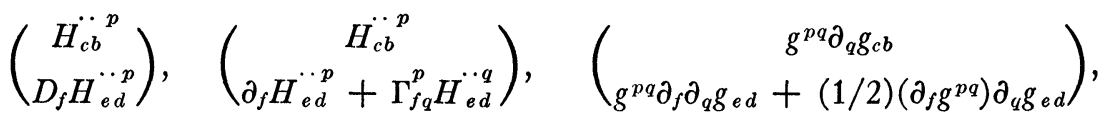

$$
\begin{aligned}
& \left(\begin{array}{c}
\partial_{p} g_{c b} \\
\partial_{f} \partial_{p} g_{\epsilon d}+(1 / 2) g_{p q}\left(\partial_{f} g^{q r}\right) \partial_{r} g_{e d}
\end{array}\right) .
\end{aligned}
$$

The last matrix shows that, unlike $m_{1}$, the dimensionality $m_{2}$ of the second normal complex of $V_{m}$ in $V_{n}$ depends not only on the nature of $g_{c b}$ but also on that of $g_{q p}$.

If $g_{q p}=g_{q p}\left(x^{r}\right)$, that is, if the complementary $V_{n-m}$ are totally geodesic in $V_{n}$ (cf. Theorem 2.1 for $\left.m_{1}=0\right)$, the matrix (4.2) reduces to

$$
\left(\begin{array}{c}
\partial_{p} g_{c b} \\
\partial_{p} \partial_{f} g_{e d}
\end{array}\right) .
$$

This matrix is of rank $m_{1}+m_{2}$, and therefore $g_{c b}$, $\partial_{f} g_{e d}$ can be expressed in terms of $x^{a}$ and $m_{1}+m_{2}$ (but not less) functions of $x^{k}$ which are independent with regard to $x^{p}$. But

$$
\begin{aligned}
g_{c b} & =g_{c b}\left(x^{a}, \rho_{1}, \cdots, \rho_{m_{1}}\right), \\
\partial_{f} g_{e d} & =\phi_{0}+\phi_{1} \partial_{f} \rho_{1}+\cdots+\phi_{m_{1}} \partial_{f} \rho_{m_{1}},
\end{aligned}
$$


where the $\phi$ 's are some functions of $x^{a}$ and the $\rho$ 's. Therefore the first and second normal complexes of $V_{m}$ in $V_{n}$ are of dimension $m_{1}$ and $m_{2}$, if and only if (4.3) is true and $\partial_{f} \rho_{1}, \cdots, \partial_{f} \rho_{m_{1}}$ are expressible in terms of $x^{a}, \rho_{1}, \cdots, \rho_{m_{1}}$, and $m_{2}$ other functions $\rho_{m_{1}+1}, \cdots, \rho_{m_{1}+m_{2}}$, which, together with $\rho_{1}, \cdots, \rho_{m_{1}}$, form $m_{1}+m_{2}$ functions independent with regard to $x^{p}$.

This being the case, we have

$$
\begin{gathered}
\partial_{p} g_{c b}=\frac{\partial g_{c b}}{\partial \rho_{1}} \partial_{p} \rho_{1}+\cdots+\frac{\partial g_{c b}}{\partial \rho_{m_{1}}} \partial_{p} \rho_{m_{1}}, \\
\partial_{p} \partial_{f} g_{e d}=\frac{\partial \partial_{f} g_{e d}}{\partial \rho_{1}} \partial_{p} \rho_{1}+\cdots+\frac{\partial \partial_{f} g_{e d}}{\partial \rho_{m_{1}+m_{2}}} \partial_{p} \rho_{m_{1}+m_{2} .} .
\end{gathered}
$$

But if $w^{a}, y^{a}, z^{a}$ are three arbitrary vectors in $V_{m}$, the vectors $y^{c} w^{b} H_{c b}^{\cdot 0 \rho}$ and $z^{f} y^{e} w^{d} D_{f} H_{e d}^{. \rho \rho}$ span the first two normal complexes of $V_{m}$ in $V_{n}$. Therefore by an argument similar to that which led to Theorem 2.2, we conclude that the first two normal complexes of $V_{m}$ at points of a fixed $V_{n-m}$ are orthogonal to a family of $V_{n-m-m_{1}-m_{2}}$ in $V_{n-m}$.

The above result can easily be extended to cover the normal complexes of higher order of $V_{m}$ in $V_{n}$; indeed we have the following two theorems.

TheOREM 4.1. In a $V_{n}$ with fundamental tensor

$$
g_{\lambda x}=\left(\begin{array}{cc}
g_{c b}\left(x^{\kappa}\right) & 0 \\
0 & g_{q p}\left(x^{r}\right)
\end{array}\right),
$$

the normal complexes of the subspaces $x^{p}=$ const. are of dimension $m_{1}, m_{2}, \cdots$ if and only if the matrices

$$
\left(\partial_{p} g_{c b}\right),\left(\begin{array}{c}
\partial_{p} g_{c b} \\
\partial_{p} \partial_{f} g_{\epsilon d}
\end{array}\right), \cdots
$$

are of ranks $m_{1}, m_{1}+m_{2}, \cdots$, respectively.

TheOREM 4.2. If a $V_{n}$ admits two families of complementary $V_{m}$ and $V_{n-m}$ and if $V_{n-m}$ are totally geodesic in $V_{n}$, then the first $l(l=1,2, \cdots)$ normal complexes of $V_{m}$ at points of any fixed $V_{n-m}$ are orthogonal to a family of $V_{n-m-m_{1}-m_{2}} \cdots_{-m_{l}}$ in $V_{n-m}$.

Cambridge, Mass. 